\title{
Can insulin response patterns predict metabolic disease risk in individuals with normal glucose tolerance?
}

\author{
Catherine A. P. Crofts ${ }^{1,2}$ (D) Kenneth Brookler ${ }^{3} \cdot$ George Henderson $^{1}$
}

Received: 30 January 2018 / Accepted: 1 February 2018 / Published online: 22 February 2018

(C) Springer-Verlag GmbH Germany, part of Springer Nature 2018

To the Editor: We commend the authors of the recent publication in Diabetologia entitled 'Glucose patterns during an oral glucose tolerance test and associations with future diabetes, cardiovascular disease and all-cause mortality rate' [1] and would encourage them to repeat their investigation using insulin response patterns in people with normal glucose tolerance. Hyperinsulinaemia is becoming well-established in the aetiology of many metabolic diseases [2], yet many people will be unaware that they are at risk. Insulin levels are posited to be elevated for many years, possibly even decades, before changes in blood glucose are noted [3, 4]. Although fasting insulin is used in many studies as a disease risk marker, it has a high coefficient of variation leading to concerns about repeatability [5]. There has been little emphasis on the use of insulin response patterns to assess the risk of metabolic disease, yet the patterns observed when tracking blood insulin levels following an OGTT may provide an earlier opportunity to assess disease risk compared with changes in blood glucose levels. Hayashi and colleagues noted that cumulative incidence of type 2 diabetes in Japanese-American men over 10 years of follow-up was between $38 \%$ and $48 \%$ in those who reached a peak insulin level at $120 \mathrm{~min}$ following a $75 \mathrm{~g}$ OGTT, which was higher than those whose insulin levels peaked at 30 or 60 min (cumulative incidence, $<16 \%$ ) [6]. However, these response patterns were based on relative insulin serum levels in a cohort that included a high proportion of participants with pre-existing impaired fasting glucose and/or impaired glucose tolerance [5]. Between 1970 and 1990, Dr J. R. Kraft collated

Catherine A. P. Crofts

ccrofts@aut.ac.nz

1 Human Potential Centre, Auckland University of Technology (AUT), Private Bag 92006, Auckland 1142, New Zealand

2 School of Interprofessional Health Studies, Auckland University of Technology (AUT), Auckland, New Zealand

3 Aerospace Medicine and Vestibular Research, Mayo Clinic, Scottsdale, AZ, USA a large database holding data from multiple-sampled OGTTs with insulin assays. Further analysis of this database suggests that up to $75 \%$ of people with normal glucose tolerance may have hyperinsulinaemia [7], but there are no longitudinal outcomes to support risk calculations. There is a distinct paucity of data on insulin response patterns and subsequent risk of cardiovascular disease and we believe the authors would advance the field with further data analysis.

Duality of interest The authors declare that there is no duality of interest associated with this manuscript.

Contribution statement $\mathrm{KB}$ was responsible for the concept and $\mathrm{CC}$ drafted the manuscript. All authors were responsible for revising it critically for important intellectual content. All authors approved the version to be published.

\section{References}

1. Hulman A, Vistisen D, Glümer C, Bergman M, Witte DR, Færch K (2018) Glucose patterns during an oral glucose tolerance test and associations with future diabetes, cardiovascular disease and allcause mortality rate. Diabetologia 61:101-107

2. Crofts C, Zinn C, Wheldon M, Schofield G (2015) Hyperinsulinemia: a unifying theory of chronic disease? Diabesity $1: 34-43$

3. Dankner R, Chetrit A, Shanik MH, Raz I, Roth J (2009) Basal-state hyperinsulinemia in healthy normoglycemic adults is predictive of type 2 diabetes over a 24-year follow-up: a preliminary report. Diabetes Care 32:1464-1466

4. Weir GC, Bonner-Weir S (2004) Five stages of evolving beta-cell dysfunction during progression to diabetes. Diabetes 53:S16-S21

5. Crofts C, Wheldon MC, Zinn C, Lan-Pidhainy X, Wolever TM, Schofield G (2017) Assessing the test-retest repeatability of insulin resistance measures: homeostasis model assessment 2 and oral glucose insulin sensitivity. J Insulin Resistance 2:9

6. Hayashi T, Boyko EJ, Sato KK et al (2013) Patterns of insulin concentration during the OGTT predict the risk of type 2 diabetes in Japanese Americans. Diabetes Care 36:1229-1235

7. Crofts C, Schofield G, Zinn C, Wheldon M, Kraft J (2016) Identifying hyperinsulinaemia in the absence of impaired glucose tolerance: an examination of the Kraft database. Diabetes Res Clin Pract 118:50-57 